\title{
La emergencia de las divas en el campo cultural latinoamericano: representaciones artísticas en la crónica modernista
}

Julieta Viu Adagio

Universidad Nacional de Rosario (UNR, Argen-

tina); CONICET.

\section{Resumen}

Las crónicas que Rubén Darío publica en la prensa chilena y Manuel Gutiérrez Nájera en la mexicana consolidan, a fines del siglo XIX, a la diva como tópico literario. Estas producciones forjan un imaginario que celebra la belleza, la armonía y la exclusividad y que por ello resulta contestatario de la concepción burguesa del arte y de la vida que rige las sociedades latinoamericanas finiseculares. En este artículo, consideramos las representaciones que los modernistas mencionados realizan de Sarah Bernhardt y Adelina Patti para analizar la emergencia del divismo, esto es, una narración donde la hegemonía de la estrella logra desplazar a un segundo plano a la obra interpretada. Contrastaremos este corpus con la crítica teatral que Paul Groussac escribe sobre Sarah Bernhardt focalizada en la actriz y sus habilidades para la interpretación.
Palabras clave

Rubén Darío, Manuel Gutiérrez Nájera, crónica, modernismo, divas 


\section{Abstract}

The emergence of divas in the Latin American cultural field: artistic representations in the modernist literary chronicle

The chronicles that Rubén Darío and Manuel Gutiérrez Nájera publish in the Chilean and Mexican presses respectively-, consolidate, at the end of the inth century, the diva as a literary topic. These productions give shape to an imaginary that celebrates beauty, harmony and exclusivity. For this reason, this imaginary rebels against the bourgeois conception of art and the life that governs Latin American societies at the end of the century. In this article, we will study the above-mentioned modernist authors' representations of Sarah Bernhardt and Adelina Patti in order to analyze the emergence of a diva-like temperament, that is, a narrative where the hegemony of the star ends up overriding the work interpreted itself. We will contrast this corpus with Paul Groussac's theater critique about Sarah Bernhardt, which focuses on the actress and her acting skills.

Resumo

\section{A emergência das divas no campo cultural latino-americano: representações artísticas na crônica modernista}

As narraçōes que publica Rúben Darío na imprensa chilena e Manuel Gutiérrez Nájera na imprensa mexicana, no final do século XIX, consolidam a diva como tópico literário. Estas produçôes forjam um imaginário que celebra a beleza, a harmonia e a exclusividade, resultando por isso uma dissidência da concepçáo burguesa da arte e da vida que rege as sociedades latino-americanas de fim do século. No presente artigo, consideramos as representaçōes que esses escritores modernistas realizam de Sarah Bernhardt e Adelina Patti, para analisar a emergência do "divismo", isto é, uma narrativa em que a hegemonia
Keywords:

Rubén Darío, Manuel

Gutiérrez Nájera, literary

chronicle, modernism, divas
Palavras-chave:

Rubén Darío, Manuel

Gutiérrez Nájera, narração, modernismo, divas 
da estrela consegui deslocar à obra interpretada a um segundo plano. Contrastaremos este corpus com a crítica teatral que Paul Groussac escreve sobre Sarah Bernhardt, centrada na atriz e suas qualidades como intérprete.

A fines del siglo XIX, destacadas figuras femeninas del teatro europeo comienzan a brindar giras artísticas por América Latina. Nombres como Eleonora Duse, María Guerrero, Sarah Bernhardt, Adelina Patti y María Tubau comienzan a escucharse en ciudades como Distrito Federal, Buenos Aires, Montevideo y Santiago de Chile. La presencia de estas personalidades distinguidas de la ópera y el teatro constituyen eventos culturales fundamentales para esas sociedades finiseculares en vías de modernización. El recibimiento chileno a Sarah Bernhardt ilustra las solemnes bienvenidas brindadas a las artistas internacionales: «la actriz fue recibida en Lota con gran iluminación, banda de músicos, ovaciones, y se le brindó acogida en los edificios del Parque» (Saavedra Molina, 1941:19). Estos programas culturales de primer orden fueron registrados por los escritores modernistas en tanto expresión de lo nuevo, lo cosmopolita, lo contemporáneo, en definitiva, como representaciones de las escenas modernas propias de la modernización en acto. Ello provoca -aquí se cifra nuestra hipótesis de lectura- la emergencia del divismo: un relato foca- lizado en la figura de la diva que desplaza $y$, en este sentido, pospone la centralidad del tratamiento y las referencias a la obra interpretada. De esta manera, surgen una serie de crónicas que forjan el imaginario de mujeres célebres, esto es, de figuras legitimadas por su belleza, su fama y su glamour. Así se consolida una galería de retratos de sublimes representantes del arte escénico.

Las crónicas que Rubén Darío publica a fines del siglo XIX en Chile resultan un punto de partida insoslayable para reflexionar sobre la aparición, en la región latinoamericana, de mujeres deseadas y admiradas por sociedades enteras. Referimos a esas figuras que logran consagrarse con auras casi míticas y que la industria cinematográfica, especialmente la hollywoodense, explotará incansablemente a lo largo del siglo XX. A meses de haber llegado al país y con apenas diecinueve años, el diario La Época de Santiago de Chile le encarga a Darío que cubra las presentaciones de una de las mayores actrices trágicas del siglo XIX que por primera vez se presentaba en el país. Entre octubre y noviembre de I886, Darío publica bajo el seudónimo Ramadés diez 
crónicas dedicadas a Sarah Bernhardt. ${ }^{\mathrm{T}}$ Motivado por el afán de novedad, de actualidad, por ese fuerte deseo modernista de conocer lo que se estaba produciendo contemporáneamente en el mundo y, especialmente en Francia, Darío escribe singulares retratos de aquella mujer admirada por personalidades que van desde Oscar Wilde a Sigmund Freud. En el Prólogo a la segunda edición de Los raros, fechado en enero de 1905 en París, el escritor afirma: «Restan la misma pasión de arte, el mismo reconocimiento de las jerarquías intelectuales, el mismo desdén de lo vulgar y la misma religión de belleza» (Darío, 20II:37). Esta serie de valores y principios que afirma sostener desde hacía nueve años (aludiendo a la primera edición del libro de 1896) ya aparecían en sus tempranas crónicas chilenas. Ello permite observar la vigencia de núcleos vertebrales que este cronista defendió con profunda convicción a lo largo de su vida: la pasión por el arte, la jerarquización intelectual, el desprecio hacia lo vulgar y el culto a la belleza. Recuperamos estos principios porque, a nuestro modo de ver, estructuran la retórica de la distinción que el cronista forja en sus crónicas sobre la diva Sarah Bernhardt.

Esta serie de crónicas se abre con $E l$ estreno de Sarah Bernhardt (Io de octubre de 1886), publicada al día siguiente del primer espectáculo, donde Ramadés se declara admirador "de la insigne artista» (1993:29) ${ }^{2}$ y, con tal confesión, explicita que abandona su deber de crítico. Gesto que encierra una de las claves de lectura: en las crónicas darianas, prima la mirada del poeta que, a diferencia de la del crítico que analiza el espectáculo, relata a partir de la seducción que experimenta. En el contexto de la división social del trabajo y su repercusión en la construcción de campos y saberes disciplinares autónomos, la escritura de Rubén Darío se diferencia de la de Paul Groussac, quien meses antes cubre, para el diario $\mathrm{La} \mathrm{Na}$ ción de Buenos Aires, la presentación de Sarah Bernhardt en dicha ciudad pero con la particularidad de posicionarse desde la perspectiva del crítico teatral. En este sentido, la declaración de Darío sobre su condición de admirador permite singularizar su producción. Pospuesta la función crítica asociada históricamente

1 Esta famosa producción, escasamente estudiada por la crítica literaria, permaneció dispersa por décadas hasta que, en 1940, fue recuperada por el crítico chileno Julio Saavedra Molina. Aunque debemos destacar que no se publicaron hasta 1988. El crítico nicaragüense Ricardo Llopesa relata los avatares de la publicación de las crónicas en el Prólogo (1993), Teatros. Prosas desconocidas de Rubén Darío (1993).

2 Las citas realizadas en este artículo corresponden a Darío (1993). Teatros. Prosas desconocidas sobre Sarah Bernhardt (edición de Ricardo Llopesa). Altea: Aitana. 
a la figura del letrado, el cronista se sumerge en el espectáculo inaugural a través del registro de sus impresiones: el "frenesí» que observa en la selecta y numerosa concurrencia, la admiración que despierta «tan excelsa artista» así como las irradiaciones de su «portentoso ingenio». Darío toma la voz de los espectadores y desde esa colectividad forja la imagen célebre. Observamos, en este punto, dos particularidades de la crónica dariana: si bien se refiere a la actriz, a su talento y a escenas de la obra —elementos clásicos de la crítica teatral—, estos aparecen de manera indirecta, es decir, a través de la respuesta del auditorio. Además, resulta notable que el público adquiere el protagonismo que tradicionalmente le corresponde a la prestigiosa voz del especialista. ${ }^{3}$

En la representación que Rubén Darío brinda de Sarah Bernhardt, es posible señalar, en primer término, ese aura estelar con que la individualiza: «su talento soberano, ha conseguido eclipsar la nombradía de la Ristori, la Pezzana, la Dussei, la Tessero, la Rossi, Savini y Calvo, es decir, la de los más egregios representantes del arte» (I993:29). La belleza, la exclusividad y la excentricidad que el joven escritor destaca del personaje la ubican en una posición de distinción que se observa claramente hacia el final de la crónica:
Y para concluir.
¿Quién es Sarah?
No sabemos decirlo.
La palabra no existe.
Sarah es lo que impele, lo que arrastra, lo que aborrece, lo que adora, lo que llora y lo que ríe.
Es mujer estatua, esfinge; es la maldad, la virtud, la firmeza indomable, la pasión que gime y se revuelve; es la soberana absoluta del arte en su más alta significación: la vida real. (1993:31)

El cronista lo explicita: no hay palabras. No resulta posible nombrar el objeto (lección aprendida de S. Mallarmé) aunque sí evocarlo a través de sensaciones. Por medio de esta estética, arriba a la imagen de una mujer sensible y seductora que pretende definir como la encarnación misma de la síntesis del arte con la vida.

3 Paul Groussac encarna indiscutiblemente, a fines del siglo XIX, el papel de crítico cultural. Paola Suárez Urtubey destaca el valor social otorgado en dicha época al especialista y lo ejemplifica con un fragmento de la nota, que reproducimos a continuación, donde La Nación anuncia el ingreso de dicho escritor como colaborador dedicado exclusivamente a la ópera y al teatro: "La Nación cuenta desde hoy con la colaboración literaria del Sr. P. Groussac, a cuyo cargo estará especialmente en nuestro diario la crítica teatral, que, en sus manos, asumirá la importancia que hace de ella en la prensa europea un alto y delicado ministerio" (2007:27). 
La diva se convierte - y allí radica la singularidad de esta escritura- en el motor de arranque de la crónica y, al mismo tiempo, en el punto de llegada. Si bien esta se vuelve una característica común a la producción dedicada a Sarah Bernhardt, destacamos la atmósfera pasional y embriagante que tiñe especialmente este texto inaugural, donde la devoción se manifiesta estéticamente a partir de la sugestión, la insinuación y la evocación, principios sostenidos por el simbolismo francés, a los que Darío apela - como veremos más adelante- para construir la imagen de la diva.

Antes de continuar con el análisis, realizamos unas breves consideraciones sobre el escrito La acción teatral. A propósito de Sarah Bernhardt de Paul Groussac, a quien el nicaragüense considera uno de sus maestros, publicado para el diario La Nación el 5 de septiembre de I886. La notable extensión del mismo, al tiempo que lo diferencia de la producción dariana, evidencia que se trata de una crónica que deviene crítica teatral. En este sentido, resulta relevante considerar que ha sido antologada en Viaje intelectual. Impresiones de naturaleza y arte I (1904), libro que aborda temas variados producto de la curiosidad del autor que tienen en común la sinceridad y el carácter informativo como se explicita en el Prefacio. Estos principios con los que el viajero francoargentino caracteriza su escritura adelantan la posición de intelectual asu- mida por el cronista. En la primera parte del texto, compuesto por comentarios de representaciones, puestas en escena y actuaciones, Paul Groussac construye un discurso riguroso, preciso y analítico que visibiliza la erudición que posee en el campo de la dramaturgia. La primacía de este registro intelectual conlleva, para ser más precisos, la censura de juicios subjetivos que sólo en contadas ocasiones deja deslizar. El contraste respecto del relato de sensaciones que Sarah despierta en Darío se vuelve aún más visible ya que Groussac abunda en comentarios y reflexiones teóricas. Al señalar el talento de la actriz, destaca que representa «obras maestras del teatro francés» (I904:278). Elogio que evidencia el parámetro eurocéntrico que ha caracterizado al ilustre director de La Biblioteca. La crónica avanza a través de disquisiciones sobre los actores, el tipo representado o la facultad innata o adquirida, entre otras cuestiones. Por momentos, recupera algún fragmento escénico como, por ejemplo, el final del cuarto acto de Las damas de las camelias para reflexionar sobre la convención teatral. De este modo Groussac recién hacia la mitad de la crónica produce una inflexión para focalizarse en Sarah:

tiene, pues, las dotes de una verdadera comedianta: se muestra dueña absoluta de sí propia y de sus efectos escénicos. Pero no mucho más que la coqueta mundana que engaña en la misma noche a diez adorado- 
res, o nuestro amigo Don Juan, al fingir admirablemente su momentánea pasión, pues la hipocresía, es decir la acción cómica, es una aptitud natural que casi todos poseemos. Procuremos, pues, analizar en pocas palabras ese talento de gran hipócrita. (I904:285, las cursivas son del original)

Esta autofiguración como especialista, dotado de facultades intelectuales que lo individualizan frente al público, le permite asumir el papel de juez cultural. Con esa «conciencia de aristos» (Rama I985:39), propia de los escritores finiseculares, Groussac se ocupa de destacar su capacidad para analizar el hecho artístico e instaura una distancia crítica para legitimar su análisis. Más clara aún resulta la afirmación "creo que esa noche era yo el único profano en el santuario» (285). Además de la construcción de esa voz narrativa, nos interesa el singular retrato que realiza de Sarah Bernhardt a partir de una descripción fisiológica que por su minuciosidad así como por la precisión terminológica presenta reminiscencias lombrosianas que se combinan con expresiones poéticas: ${ }^{4}$

La cabeza no es regularmente hermosa, si bien toda ella modelada en fracciones expresivas, que forman un conjunto de extraña seducción. La fisonomía está hecha de relieves y méplats: ninguna redondez. La curva netamente hebraica del perfil se interrumpe de golpe sobre el duro relieve del labio corto, desdeñoso, con los surcos de la ironía marcadísimos. Las alas de la nariz se dilatan fácilmente en los momentos de pasión; durante los raptos de ira, el músculo piramidal se hincha agresivo, entre las cejas fruncidas. El párpado espeso cubre los admirables ojos de agua marina o turquesa, pues su color parece mudable como el del mar... (1904:286, las cursivas son del original)

Este retrato evidencia una tensión entre la descripción de la fisonomía, marcada por la precisión nominativa que le otorga cientificidad al discurso, y las expresiones metafóricas que distienden la densa enumeración anterior. Focalizado en la actriz, Groussac destaca que posee «uno de los más perfectos instrumentos vocales que en el teatro haya resonado" (I904:288) aunque presenta debilidades en la destreza gestual: «Muchos otros detalles, criticables por su audacia o repetición, podrían señalarse; pero me limitaré al más grave y general, que consiste en la monotonía del tipo representado» (290).

4 Con la expresión "reminiscencias lombrosianas" referimos al discurso positivista criminológico, desarrollado entre otros por el médico César Lombroso, quien a fines del siglo XIX había establecido una clasificación de los criminales a partir de rasgos físicos que permitían identificarlos. 
La crónica concluye — no podía ser de otra manera- con un juicio crítico: «Sarah Bernhardt no logró nunca realizar a la perfección las primeras figuras; pero nadie supo como ella prestar a las segundas color intenso y relieve eficaz» (290).

Esta breve referencia a Paul Groussac ilumina la distancia que media entre el estudio riguroso de éste y la primacía de la impresión subjetiva en Rubén Darío. Aunque motivado por el mismo tema, el nicaragüense asume una perspectiva distinta que le permite centrarse en el entusiasmo y la seducción despertados por un ser tan sublime. La mayor diferencia entre estos escritores radica en el modo de representar a la protagonista: Rubén Darío forja la imagen de la diva, mientras que Paul Groussac trabaja con la actriz. Edgar Morin, en el clásico libro Las estrellas del cine, señala que la estrella posee un plus respecto de la actriz ya que aquella trasciende a ésta: «La estrella es el actor o la actriz que absorbe una parte de la sustancia heroica -es decir, divinizada y mítica- de los héroes de cine, y que, recíprocamen- te, enriquece esta sustancia mediante un aporte que le es propio» (1966:45). Este análisis resulta operativo para reflexionar sobre el proceso de divinización capaz de convertir a una actriz en una diosa y, por ende, se vuelve pertinente para abordar a la Sarah dariana, quien deviene ese ser mixto producto de la imposibilidad de discernir entre la actriz y su personaje. Cuando ella representa Adriana Lecouvreur, Darío afirma: «Nosotros no hemos visto a la actriz esa, para quien fue escrita Adriana; pero estamos seguros, y abonados por criterios bien fundados, de que Sarah en las tablas de cualquier teatro del mundo, interpreta, ilumina, mejora, la creación de Scribe y Legouvé» (1993:4I, las cursivas son del original).

Una característica de Sarah Bernhardt que Rubén Darío recupera constantemente es su singular belleza. Si atendemos a los adjetivos que emplea para nombrarla, observamos que representan principalmente refinamiento y excelencia: es la «soberana absoluta del arte» (37), «mujer sensible y seductora» (3I) de un «temperamento

$5 \mathrm{El}$ crítico argentino Enrique Foffani desarrolla el sentido que la belleza tuvo para los modernistas en tanto categoría secular: "La belleza en tanto que territorio estético de la fantasía poética en un sentido amplio y no sólo en el estricto del género, opera en el modernismo hispanoamericano como una presencia disidente que debe enfrentar los valores impuestos $y$, por ende, defendidos por una modernidad rastacuera y servil (...). Contra el baño de utilitarismo del que ya no pueden sustraerse todas las cosas, la belleza es el ámbito por antonomasia de la resistencia del artista en su intento por sobrevivir al tiempo presente percibido como una crisis histórica radical, preñada de angustia y de un sentimiento amargo de la existencia. La belleza repudia la moral filistea del capitalismo y contrapone la moral sublime del arte" (2007:14, las cursivas son del original). 
excepcional» (35). Aclaramos que la belleza presenta un doble sentido: además de referir a las cualidades físicas de la diva, supone la encarnación del ideal artístico $y$, en este sentido, vehiculiza una crítica a la sociedad materialista finisecular. ${ }^{5}$ Darío ratifica que se trata de un ser extraordinario y, de este modo, visibiliza otro rasgo de la diva que es la exclusividad: «ella es todo» (3I), «Sarah es única» (30), expresa hiperbólicamente el cronista. Si bien por momentos refiere a la actuación, como explicita en reiteradas ocasiones, decide posponer los comentarios sobre la obra para abocarse a la diva como vemos en el comienzo de Adriana Lecouvreur: «No queremos hablar de la obra antes de referirnos nuevamente a esa mujer: Sarah, para la cual se han hecho dramas como vestidos» (39). En síntesis, allí se forja una retórica reivindicativa, exuberante y profusamente elogiosa.

La apropiación de la figura de la diva y sus representaciones «en un exquisito francés» (Ballón Aguirre, 20I2:4I4) visibiliza la singular apertura que Rubén
Darío tuvo respecto a las novedades así como de las expresiones cosmopolitas. El cronista destaca y, por tanto, valora a Sarah Bernhardt en tanto mujer ilustre, célebre, insigne y sublime. Aunque podríamos afirmar que lo que más admira es que «todos los vicios y las virtudes, todas las debilidades del alma, todos los arranques generosos de un corazón hidalgo, tienen en el semblante, la voz y la actitud de Sarah sus medios más apropiados de expresión» (1993:36). En definitiva, observa a Sarah como una mujer que va más allá del sujeto moderno, un ser pasional gobernado por sus sentimientos. En ese gesto ciframos el rechazo que el modernismo manifiesta hacia la modernidad entendida como realización del proyecto normativo de la Ilustración especialmente en tres factores: el desarrollo de la ciencia, la moralidad y el arte de acuerdo a sus lógicas internas y de manera autónoma. Se trata del proceso de racionalización que moldea la subjetividad bajo imperativos económicos y administrativos. ${ }^{6}$

6 Jurgen Habermas sostiene que "el proyecto de modernidad formulado en el siglo XVIII por los filósofos de la llustración consistió en sus esfuerzos para desarrollar una ciencia objetiva, una moralidad y leyes universales y un arte autónomo acorde a su lógica interna" (1983:28). La búsqueda de institucionalización que se corresponde con la creación de un discurso científico así como de teorías sobre la moralidad, la jurisprudencia y las creaciones artísticas estaría bajo el dominio de expertos o especialistas. Estas reflexiones sobre el arte y la cultura europea resultan iluminadoras para reflexionar sobre el modernismo latinoamericano ya que, como ha explicitado el crítico colombiano Rafael Gutiérrez Girardot (2004), si bien no resulta posible hablar de la existencia de una clase burguesa en América Latina sí podemos encontrar en la sociedad finisecular una mentalidad burguesa basada en principios racionales y capitalistas. 
Otro caso ejemplar para visualizar la aparición de las divas en el campo cultural latinoamericano lo constituyen las crónicas que Manuel Gutiérrez Nájera publica para dos de los diarios más importantes de México, El Universal y El partido liberal, dedicadas a la soprano italiana Adelina Patti. ${ }^{7}$ A diferencia del caso anterior, la retórica de la distinción se singulariza, en este caso, por la construcción de un discurso que escenifica la distancia. Mientras que el escritor nicaragüense se limita a representar exclusivamente a la diva, el cronista mexicano pone en escena y, de este modo, resalta las diferencias entre el arte culto de la soprano y las expresiones populares. Antes de abocarnos al análisis de estas producciones, recuperamos caracterizaciones que el propio Gutiérrez Nájera brinda sobre distintas manifestaciones culturales para observar tensiones que operan a fines del siglo XIX sobre las prácticas artísticas. En la crónica Espectáculos populares, aborda lo que considera diversiones vulgares: por un lado, refiere a los «teatritos suciamente improvisados en las plazas» (2003:I08) a los que asisten principalmente «mujeres desvergonzadas» (I09). Se trata de espec- táculos de gran concurrencia pero que dan «a la ciudad el aspecto de un villorio en día de feria» (I08). Por otro lado, alude a las corridas de toros, ese espacio donde «el hijo lanza la palabra más soez junto a su padre, sin que éste dé el menor signo de enojo» (IO9). Gutiérrez Nájera, como puede deducirse, observa allí solo expresiones de atraso y decadencia, uno de los extremos de la oferta cultural, que se opone a ese acontecimiento aristocrático que es el teatro donde el público asiste vestido de etiqueta. La representación de esta otra escena la encontramos justamente en el escrito titulado Adelina Patti, donde el cronista asiste a la función de ópera y al sentarse en la luneta, observa: «No hay asiento que no esté ocupado, y ocupado por un frac o por un traje de seda. La pobreza, refunfuñando, quedó afuera. Aquí están todos los diamantes, todas las camelias» (2003:II2). De ambas caracterizaciones, queda claro que la muchedumbre se divierte en las plazas mientras que el público selecto ("grandes señoras, banqueros, aristócratas» 2003:II2) asiste al teatro. Estas críticas cargadas de valoraciones sociales que aparecen representadas en las crónicas

7 Las reflexiones que presentamos en este apartado son producto no sólo de la lectura de las crónicas dedicadas a Adelina Patti sino de un conjunto más amplio de crónicas sobre el teatro entre las que se encuentran: Espectáculos populares, Adelina Patti, Sarah Bernhardt. Carta a Justo Sierra, en La música y el instante. Crónicas (2003); Una cita y El viejo invierno, en Narraciones (antología) (2003); Oír a la Patti, A la señora Adelina Patti y Ya no canta la Patti, en Obras VII. Crónicas y artículos sobre teatro, $V(1890-1892)$ (1990). 
iluminan la concepción que los escritores modernistas tuvieron del teatro. El crítico peruano José Ballón Aguirre destaca el carácter antipopular del mismo: «en el siglo XIX latinoamericano el teatro era de por sí un evento cultural caracterizado por su elitismo y su poder de exclusión social» (20I2:440) ya que resultaba accesible para un público restringido no solo por el alto costo sino también porque las obras se representaban en su idioma original. La tensión entre lo culto y lo popular se manifiesta, más allá de las posibilidades económicas, en el conocimiento de lenguas extranjeras.

Cuando la soprano italiana visita por primera vez México, Gutiérrez Nájera escribe Adelina Patti, donde festeja que se haya podido efectivizar el concierto ya que, como menciona al comienzo, a fines del año anterior los mexicanos fueron estafados porque se les vendieron boletos falsos. Al recuperar esta anécdota, Gutiérrez Nájera describe la entrada triunfal de la artista: "Todos los cuellos se alargan, todos los anteojos se dirigen al escenario entre un murmullos como de palmas que se agitan alrededor del carro de la joven vencedora. ¡Ahí está Adelina!» (2003:II3). Esta «divina maestra de ruiseñores» (III) ha logrado conmover al público y al cronista de singular manera:

Ahora solo pensamos, los admiradores de Adelina, en batirnos à outrance con el que no aplauda, con el que no se ponga de pie esta noche cuando ella salga al escenario. Las ideas salen de mi cerebro atropellándose, gritando hurras, como salimos anoche del teatro. Miro, reflejado en el papel, el rostro de Sofía Scalchi y creo que su bronca me pregunta: ¿Y yo? Pero, ¡Imposible apartar la vista de Adelina! Nos ha puesto de rodillas y no podemos levantarnos. (II3)

Este fragmento interesa porque el cronista individualiza a la estrella pero, además, debido a que pone en escena la urgencia del pensamiento, la aceleración del tiempo, esto es, la intensidad con que se experimenta la modernidad. Ello nos recuerda al José Martí de Versos libres (I878-I882) asediado por imágenes que le salen a borbotones de la herida. Se cifran allí dos percepciones modernas: la urgencia propia de la crónica, es decir, de la escritura para la prensa periódica y, al mismo tiempo, la intensidad y el fervor ante esa experiencia cosmopolita que supone asistir al concierto de la soprano italiana. Este fragmento produce un corte en la crónica al dar comienzo a una larga serie de halagos expresados enfáticamente sobre la hermosura de sus ojos y, principalmente, de su voz así como de su juventud. El lenguaje se carga de figuras retóricas y abandona la intención informativa:

Culturas I3 - Debates y perspectivas de un mundo en cambio · I7I 
Imaginaos un perfume que se oye. Yo jamás lo había imaginado, pero lo he sentido. Imaginaos una evasión de mariposas de cristal que chocan sus alitas en el aire, que rozan nuestros oídos, que se detienen en nuestros labios, que se ríen, que se quejan, y que no extinguen, ique no mueren, que se van! Al oírla, se desearía tener a mano una de esas redes con que los niños cazan mariposas (...) Esa voz hace frisos de la Alhambra con moléculas de aire. Es un encaje que canta. (II4-IIS)

Con estas expresiones poéticas, el escritor transita las últimas partes de la crónica y, hacia el final, explicita — podríamos pensar, simulando oír al editor o a los reporteros-: "No me habléis de sus joyas, son notas que ella ha cuajado y convertido en collares, en aderezos, en rivières. De alhajas y de trajes hablaremos otra vez» (II6, las cursivas son del original). La priorización de la expresión literaria ante la palabra facticia del periodismo, rasgo común a los autores modernistas, visibiliza la posición del escritor frente al pragmatismo reinante de la sociedad materialista finisecular $y$, en este sentido, la crónica ilustra el papel que los modernistas asumieron como guardianes del ideal y de la belleza.
La crónica $A$ la señora Adelina Patti, publicada en El Partido Liberal el 24 de enero de 1890 bajo el aristocrático seudónimo El duque Job, resulta representativa de esa posición intermedia que asume Gutiérrez Nájera entre el crítico especializado y el admirador. La particularidad es que se trata de un texto escrito ante el recital brindado por la soprano el 22 de enero a beneficio del Hospicio de Pobres, la Escuela Industrial de Huérfanos y la Casa Amiga de la Obrera. Es una respuesta a la nota titulada $A l$ público que salió el día anterior en el diario El Universal, donde la artista explicita los motivos de su actuación: la generosidad y el cariño del público. ${ }^{8}$ Este contexto que da origen a la crónica permite entender, en primer lugar, el punto de vista desde el cual está escrita (el cronista habla en primera persona, como lo hace generalmente, pero en nombre - y esto es lo singular- de los humildes); en segundo lugar, la forma epistolar que asume y que impone un tono íntimo y confesional; por último, el valor de la beneficencia como motor de la escritura. Estos rasgos la diferencian notablemente del resto de las crónicas que Gutiérrez Nájera dedica a Adelina Patti. Detengámonos en el comienzo: «Esas manitas que todavía no saben aplaudir,

8 Elvira López Aparicio reproduce parte de la nota que Adelina Patti hizo publicar: "cedo gustosa la parte que como artista me corresponde en esta noche, según mi contrato con la empresa. Corta será la dádiva, pero grande la voluntad y el afecto con que la agradece en testimonio de amor a México, quien agradece y nunca olvida la benevolencia de este ilustrado público" (1990:10). 
son, señora, las que mejor os han aplaudido. Esas lágrimas que la gratitud arranca a las pupilas de las pobres madres, son, señora, vuestros diamantes más valiosos. Nos parecéis ahora más hermosa. Antes habíais cantado como ruiseñor. Anoche habéis cantado como ángel» (I990:IO). Esta crónica asume el rol de mediadora entre la artista (la "señora») y los beneficiados por el show, incapaces de valorar un espectáculo culto como es la ópera («esas manitas que todavía no saben aplaudir»). El duque Job, que confiesa no haber asistido a la función pero que retiene la voz en su memoria, convierte a Patti en un ángel, ángel que pronto será «una hada muy bella y muy buena» (II) con un canto dulce y suave. Allí, se visibiliza que la verdadera protagonista de la crónica es la voz de este ser mágico y armónico. Por supuesto, no olvidamos sino que destacamos el valor supremo que la armonía tuvo para el modernismo. La artista italiana es representada por su sensibilidad, delicadeza, dulzura y elegancia: «esa voz que despierta ternuras en el alma; que va abriendo, con besos, los ojitos de todos nuestros amores dormidos; que vuela y que deslumbra, como colibrí; voz siempre fresca, siempre joven, siempre virgen» (IO). Estos valores contrastan con los ausentes, aquellas personas hacia las que está dirigida la acción benéfica:

pero las perlas [la voz] caían, no en el mármol de los tocadores elegantes, no en las copas de cristal bohemio erguidas sobre la mesa del festín, no en la seda de los trajes ostentosos ni en el alabastrino cuello de las damas, sino en las cunas toscas de los niños indigentes, en los catres de los hospitales, en la mesa, sin mantel ni pan, de los menesterosos; y ${ }_{i}$ cosa rara! Esas perlas sonaban mejor al caer sobre la estera del enfermo pobre. (II)

Adelina Patti, divinizada, en las distintas crónicas, por los ideales estéticos que representa, resulta un ser adorable no solo por esa cualidad vocal inigualable sino también por ser un alma caritativa. La retórica (en sentido de adorno como de estructura discursiva lógica) se tensiona, como ilustra el fragmento, entre el lujo y la austeridad aunque al priorizar el acto de beneficencia, el cronista armoniza esa brecha que parecería ser insalvable: la riqueza cultural frente a la pobreza económica; la elegancia del artista frente a la indigencia social; esa voz noble frente a los incultos de cunas toscas. Tal vez podríamos sintetizarlo, desde la perspectiva que Gutiérrez Nájera presenta, como la voz frente a los que no tienen voz: «Señora, en nombre de los infelices —más infelices hoy porque ya no podrán oíros nunca- ¡muchas gracias!» (I2), concluye el texto. De esta manera, se visibiliza una doble función: el cronista como representante de los sectores de la alta sociedad le trasmite a la diva la gratitud de hombres y mujeres pobres que recibirán su ayuda 
y, al mismo tiempo, anoticia a la sociedad mexicana del acto de caridad.

Mientras que Rubén Darío construye a Sarah Bernhardt como diva sin aludir a la nominación sino justamente mediante retratos sugerentes que devuelven una imagen de un ser excepcional e inalcanzable; Manuel Gutiérrez Nájera refiere de manera directa a Adelina Patti como diva. Resulta notable que mientras aquél opera por omisión, esto es, solo ve lo bello (una estrella y un público que se corresponden); el mexicano construye una retórica de la distinción a partir de contrastes como analizamos por ejemplo en $A$ la señora Adelina Patti. Estas crónicas publicadas para un lector culto valoran las expresiones artísticas de fama internacional y, en este sentido, cosmopolitas en detrimento de las representaciones locales y populares. Lo universal se asocia a la expresión por antonomasia de lo moderno y lo local a lo retrasado. Los modos de autorización de los cronistas también dan cuenta de la identificación de éstos con el público al que se dirigen: ambos asisten regularmente a la ópera y comparten ideales artísticos, conocimientos de literatura, música y teatro. $\mathrm{Si}$ apelamos a la perspectiva simbolista de
S. Mallarmé y considerando la distancia que establecen respecto al parnasianismo, podemos concluir que Rubén Darío responde más cabalmente al simbolismo ya que realiza una fuerte apuesta por el potencial visual del lenguaje, es decir, va rodeando el objeto (la diva) de modo que el lector adivine poco a poco la figura; en cambio, las crónicas de Manuel Gutiérrez Nájera se encuentran más próximas a los parnasianos porque toma el objeto por entero y lo expone sin más. ${ }^{9}$ Mencionadas las diferencias sustanciales, rescatamos el punto en común más relevante: el divismo que aparece representado por los autores mencionados resulta contestatario a la concepción burguesa de la vida producto del incipiente régimen capitalista de las principales ciudades latinoamericanas. Este signo contestatario obedece, entre otras cuestiones, a los valores y principios enarbolados: el lugar central otorgado al arte como desafío a las prácticas utilitarias; la expresión sublime como bandera contra el materialismo reinante; lo excepcional de las representaciones artísticas frente a la vulgaridad de la realidad cotidiana; el ser pasional frente al homo economicus. Paradójicamente el divismo se sustenta en uno de los pila-

9 Referimos a los postulados estéticos de una de las corrientes poéticas francesas más importantes del siglo XIX, entre los que se destacan -siguiendo la historización planteada en Parnaso y simbolismo (1948)- Leconte de Lisle, Th. Banville, Catulle Mendès, Sully Prudhomme y José María de Heredia. 
res de la cosmovisión burguesa como es el individualismo. Ambos exaltan al sujeto individual en detrimento de las nominaciones colectivas. Rafael Gutiérrez Girardot, en «El arte en la sociedad burguesa moderna», sostiene que ésta se rige por «el egoísmo como principio general, las dependencias recíprocas, el interés propio y el principio de utilidad» (2004:45). Así como la burguesía concibe al ciudadano como sujetos privados motivados por la realización de intereses particulares, el estrellato demanda a las divas la construcción de una firma, de un nombre propio capaz de designar a un sujeto inconfundible, inimitable y altamente singular.

\section{Referencias bibliográficas}

- BALLÓN AGUIRRE, J. (2012). Martí y Darío ante América y Europa. Textos y contextos contrarios. México: UNAM.

- DARío, R. (1993). Teatros. Prosas desconocidas sobre Sarah Bernhardt (edición de Ricardo Llopesa). Altea: Aitana.

-_-_ (2011). Prólogo. En Los raros (p. 37). Buenos Aires: Losada.

- FOFFANI, E. (2007). Introducción. En Foffani, E. (Comp.). La protesta de los cisnes (pp. 13-43). Buenos Aires: Katatay.

- GROUSSAC, P. (1904). Viaje intelectual. Impresiones de naturaleza y arte I. Madrid: Librería General de Victoriano Suárez.

- GUTIÉRREZ GIRARDOT, R. (2004). Modernismo. México: Fondo de Cultura Económica.

- GUTIÉRREZ NÁJERA, M. (1990). Obras VII. Crónicas y artículos sobre teatro $V(1890-1892)$. México: UNAM.

-__ (2003). La música y el instante. Crónicas. Caracas: Biblioteca Ayacucho.

- HABERMAS, J. (1983). La modernidad, un proyecto incompleto. En Foster, H. (ed.), La posmodernidad (pp. 19-36). Barcelona: Kairós.

- LLOPESA, R. (1993). Prólogo. En Darío, R., Teatros. Prosas desconocidas sobre Sarah Bernhardt (pp. 9-25). Altea: Aitana. - LÓPEZ APARICIO, E. (1990). Introducción. En Gutiérrez Nájera, 
M., Obras VII. Crónicas y artículos sobre teatro V (1890-1892) (pp. XLIV-LXXII). México: UNAM.

- MARTINO, P. (1948). Parnaso y Simbolismo. Buenos Aires: EI Ateneo.

- MORIN, E. (1966). Las estrellas del cine. Buenos Aires: Eudeba. - RAMA, A. (1985). Las máscaras democráticas del modernismo. Montevideo: Fundación Ángel Rama.

- SAAVEDRA MOLINA, J. (1941). Rubén Darío y Sarah Bernhardt. En Anales de la Universidad (pp. 17-45), Santiago de Chile. - SUÁREZ URTUBEY, P. (2007). Estudio preliminar. En Groussac, P., Críticas sobre música (pp. 15-46). Buenos Aires: Biblioteca Nacional. 